\title{
Asymptotic Behaviour of Eigenvalues and Eigenfunctions of a Sturm-Liouville Problem with Retarded Argument
}

\author{
Erdoğan Şen, ${ }^{1,2}$ Jong Jin Seo, ${ }^{3}$ and Serkan Araci ${ }^{4}$ \\ ${ }^{1}$ Department of Mathematics, Faculty of Arts and Science, Namik Kemal University, 59030 Tekirdağ, Turkey \\ ${ }^{2}$ Department of Mathematics Engineering, Istanbul Technical University, Maslak, 34469 Istanbul, Turkey \\ ${ }^{3}$ Department of Applied Mathematics, Pukyong National University, Busan 608-737, Republic of Korea \\ ${ }^{4}$ Department of Mathematics, Faculty of Science and Arts, University of Gaziantep, 27310 Gaziantep, Turkey
}

Correspondence should be addressed to Jong Jin Seo; seo2011@pknu.ac.kr

Received 18 November 2012; Accepted 1 March 2013

Academic Editor: Suh-Yuh Yang

Copyright (C) 2013 Erdoğan Şen et al. This is an open access article distributed under the Creative Commons Attribution License, which permits unrestricted use, distribution, and reproduction in any medium, provided the original work is properly cited.

In the present paper, a discontinuous boundary-value problem with retarded argument at the two points of discontinuities is investigated. We obtained asymptotic formulas for the eigenvalues and eigenfunctions. This is the first work containing two discontinuities points in the theory of differential equations with retarded argument. In that special case the transmission coefficients $\delta=\gamma=1$ and retarded argument $\Delta \equiv 0$ in the results obtained in this work coincide with corresponding results in the classical Sturm-Liouville operator.

\section{Introduction}

Delay differential equations arise in many areas of mathematical modelling, for example, population dynamics (taking into account the gestation times), infectious diseases (accounting for the incubation periods), physiological and pharmaceutical kinetics (modelling, for example, the body's reaction to $\mathrm{CO}_{2}$, and so forth, in circulating blood) and chemical kinetics (such as mixing reactants), the navigational control of ships and aircraft, and more general control problems. Also, differential equations and nonlinear differential equations have been studied by many mathematicians in several ways for a long time cf. [1-20].

Boundary value problems for differential equations of the second order with retarded argument were studied in [5$10,13-16]$, and various physical applications of such problems can be found in [6].

In the papers [13-16], the asymptotic formulas for the eigenvalues and eigenfunctions of a discontinuous boundary value problem with retarded argument and a spectral parameter in the boundary conditions were derived. In spite of their being already a long years, these subjects are still today enveloped in an aura of mystery within scientific community although they have penetrated numerous mathematical field.

The asymptotic formulas for the eigenvalues and eigenfunctions of the Sturm-Liouville problem with the spectral parameter in the boundary condition were obtained in [1720].

In this paper we study the eigenvalues and eigenfunctions of a discontinuous boundary value problem with retarded argument. Namely, we consider the boundary value problem for the differential equation

$$
y^{\prime \prime}(x)+q(x) y(x-\Delta(x))+\lambda y(x)=0
$$

on $\left[0, h_{1}\right) \cup\left(h_{1}, h_{2}\right) \cup\left(h_{2}, \pi\right]$, with boundary conditions

$$
\begin{aligned}
& y(0) \cos \alpha+y^{\prime}(0) \sin \alpha=0, \\
& y(\pi) \cos \beta+y^{\prime}(\pi) \sin \beta=0,
\end{aligned}
$$


and transmission conditions

$$
\begin{gathered}
y\left(h_{1}-0\right)-\delta y\left(h_{1}+0\right)=0, \\
y^{\prime}\left(h_{1}-0\right)-\delta y^{\prime}\left(h_{1}+0\right)=0, \\
y\left(h_{2}-0\right)-\gamma y\left(h_{2}+0\right)=0, \\
y^{\prime}\left(h_{2}-0\right)-\gamma y^{\prime}\left(h_{2}+0\right)=0,
\end{gathered}
$$

where the real-valued function $q(x)$ is continuous in $\left[0, h_{1}\right) \cup$ $\left(h_{1}, h_{2}\right) \cup\left(h_{2}, \pi\right]$ and has finite limits

$$
q\left(h_{1} \pm 0\right)=\lim _{x \rightarrow h_{1} \pm 0} q(x), \quad q\left(h_{2} \pm 0\right)=\lim _{x \rightarrow h_{2} \pm 0} q(x),
$$

the real-valued function $\Delta(x) \geq 0$ continuous in $\left[0, h_{1}\right) \cup$ $\left(h_{1}, h_{2}\right) \cup\left(h_{2}, \pi\right]$ and has finite limits

$$
\Delta\left(h_{1} \pm 0\right)=\lim _{x \rightarrow h_{1} \pm 0} \Delta(x), \quad \Delta\left(h_{2} \pm 0\right)=\lim _{x \rightarrow h_{2} \pm 0} \Delta(x) ;
$$

if $x \in\left[0, h_{1}\right)$ then $x-\Delta(x) \geq 0$; if $x \in\left(h_{1}, h_{2}\right)$ then $x-\Delta(x) \geq h_{1}$; if $x \in\left(h_{2}, \pi\right)$ then $x-\Delta(x) \geq h_{2} ; \lambda$ is a real spectral parameter; $h_{1}, h_{2}, \alpha, \beta, \delta, \gamma \neq 0$ are arbitrary real numbers such that $0<h_{1}<h_{2}<\pi$ and $\sin \alpha \sin \beta \neq 0$.

It must be noted that some problems with transmission conditions which arise in mechanics (thermal condition problem for a thin laminated plate) were studied in [20].

Let $w_{1}(x, \lambda)$ be a solution of (1) on $\left[0, h_{1}\right]$, satisfying the initial conditions

$$
w_{1}(0, \lambda)=\sin \alpha, \quad w_{1}^{\prime}(0, \lambda)=-\cos \alpha .
$$

The conditions (10) define a unique solution of (1) on $\left[0, h_{1}\right]$ ([5], page 12).

After defining the above solution, then we will define the solution $w_{2}(x, \lambda)$ of $(1)$ on $\left[h_{1}, h_{2}\right]$ by means of the solution $w_{1}(x, \lambda)$ using the initial conditions

$$
w_{2}\left(h_{1}, \lambda\right)=\delta^{-1} w_{1}\left(h_{1}, \lambda\right), \quad w_{2}^{\prime}\left(h_{1}, \lambda\right)=\delta^{-1} w_{1}^{\prime}\left(h_{1}, \lambda\right) .
$$

The conditions (11) define a unique solution of $(1)$ on $\left[h_{1}, h_{2}\right]$.

After describing the above solution, then we will give the solution $w_{3}(x, \lambda)$ of $(1)$ on $\left[h_{2}, \pi\right]$ by means of the solution $w_{2}(x, \lambda)$ using the initial conditions

$$
w_{3}\left(h_{2}, \lambda\right)=\gamma^{-1} w_{2}\left(h_{2}, \lambda\right), \quad w_{3}^{\prime}\left(h_{2}, \lambda\right)=\gamma^{-1} w_{2}^{\prime}\left(h_{2}, \lambda\right) .
$$

The conditions (12) define a unique solution of (1) on $\left[h_{2}, \pi\right]$.

Consequently, the function $w(x, \lambda)$ is defined on $\left[0, h_{1}\right) \cup$ $\left(h_{1}, h_{2}\right) \cup\left(h_{2}, \pi\right]$ by the equality

$$
w(x, \lambda)= \begin{cases}w_{1}(x, \lambda), & x \in\left[0, h_{1}\right), \\ w_{2}(x, \lambda), & x \in\left(h_{1}, h_{2}\right), \\ w_{3}(x, \lambda), & x \in\left(h_{2}, \pi\right],\end{cases}
$$

is a solution of $(1)$ on $\left[0, h_{1}\right) \cup\left(h_{1}, h_{2}\right) \cup\left(h_{2}, \pi\right]$, which satisfies one of the boundary conditions and four transmission conditions.
Lemma 1. Let $w(x, \lambda)$ be a solution of (1) and $\lambda>0$. Then the following integral equations hold:

$$
\begin{aligned}
& w_{1}(x, \lambda)= \sin \alpha \cos s x-\frac{\cos \alpha}{s} \sin s x \\
&-\frac{1}{s} \int_{0}^{x} q(\tau) \sin s(x-\tau) w_{1}(\tau-\Delta(\tau), \lambda) d \tau \\
&(s=\sqrt{\lambda}, \lambda>0)
\end{aligned}
$$

$$
\begin{aligned}
w_{2}(x, \lambda)= & \frac{1}{\delta} w_{1}\left(h_{1}, \lambda\right) \cos s\left(x-h_{1}\right) \\
& +\frac{w_{1}^{\prime}\left(h_{1}, \lambda\right)}{s \delta} \sin s\left(x-h_{1}\right) \\
& -\frac{1}{s} \int_{h_{1}}^{x} q(\tau) \sin s(x-\tau) w_{2}(\tau-\Delta(\tau), \lambda) d \tau \\
w_{3}(x, \lambda)= & \frac{1}{\gamma} w_{2}\left(h_{2}, \lambda\right) \cos s\left(x-h_{2}\right) \\
& +\frac{w_{2}^{\prime}\left(h_{2}, \lambda\right)}{s \gamma} \sin s\left(x-h_{2}\right) \quad(15) \\
& -\frac{1}{s} \int_{h_{2}}^{x} q(\tau) \sin s(x-\tau) w_{3}(\tau-\Delta(\tau), \lambda) d \tau \\
& \quad(s=\sqrt{\lambda}, \lambda>0) .
\end{aligned}
$$

Proof. To prove this lemma, it is enough to substitute $-s^{2} w_{1}$ $(\tau, \lambda)-w_{1}^{\prime \prime}(\tau, \lambda),-s^{2} w_{2}(\tau, \lambda)-w_{2}^{\prime \prime}(\tau, \lambda)$, and $-s^{2} w_{3}(\tau, \lambda)-$ $w_{3}^{\prime \prime}(\tau, \lambda)$ instead of $-q(\tau) w_{1}(\tau-\Delta(\tau), \lambda),-q(\tau) w_{2}(\tau-\Delta(\tau), \lambda)$, and $-q(\tau) w_{3}(\tau-\Delta(\tau), \lambda)$ in the integrals in (14), (15), and (16), respectively, and integrate by parts twice.

Theorem 2. The problem (1)-(7) can have only simple eigenvalues.

Proof. Let $\tilde{\lambda}$ be an eigenvalue of the problem (1)-(7) and

$$
\tilde{y}(x, \tilde{\lambda})= \begin{cases}\tilde{y}_{1}(x, \tilde{\lambda}), & x \in\left[0, h_{1}\right), \\ \tilde{y}_{2}(x, \tilde{\lambda}), & x \in\left(h_{1}, h_{2}\right), \\ \tilde{y}_{3}(x, \tilde{\lambda}), & x \in\left(h_{2}, \pi\right]\end{cases}
$$

be a corresponding eigenfunction. Then, from (2) and (10), it follows that the determinant

$$
W\left[\tilde{y}_{1}(0, \tilde{\lambda}), w_{1}(0, \tilde{\lambda})\right]=\left|\begin{array}{cc}
\tilde{y}_{1}(0, \tilde{\lambda}) & \sin \alpha \\
\tilde{y}_{1}^{\prime}(0, \tilde{\lambda}) & -\cos \alpha
\end{array}\right|=0,
$$

and, by Theorem 2.2.2, in [5] the functions $\tilde{y}_{1}(x, \tilde{\lambda})$ and $w_{1}$ $(x, \tilde{\lambda})$ are linearly dependent on $\left[0, h_{1}\right]$. We can also prove that the functions $\tilde{y}_{2}(x, \tilde{\lambda})$ and $w_{2}(x, \tilde{\lambda})$ are linearly dependent on 
$\left[h_{1}, h_{2}\right]$ and $\tilde{y}_{3}(x, \tilde{\lambda})$ and $w_{3}(x, \tilde{\lambda})$ are linearly dependent on $\left[h_{2}, \pi\right]$. Hence

$$
\tilde{y}_{i}(x, \tilde{\lambda})=K_{i} w_{i}(x, \tilde{\lambda}) \quad(i=1,2,3),
$$

for some $K_{1} \neq 0, K_{2} \neq 0$, and $K_{3} \neq 0$. We must show that $K_{1}=$ $K_{2}$ and $K_{2}=K_{3}$. Suppose that $K_{2} \neq K_{3}$. From the equalities (6) and (19), we have

$$
\begin{aligned}
\tilde{y}\left(h_{2}\right. & -0, \widetilde{\lambda})-\gamma \widetilde{y}\left(h_{2}+0, \widetilde{\lambda}\right) \\
& =\widetilde{y_{2}}\left(h_{2}, \widetilde{\lambda}\right)-\gamma \widetilde{y_{3}}\left(h_{2}, \tilde{\lambda}\right) \\
& =K_{2} w_{2}\left(h_{2}, \widetilde{\lambda}\right)-\gamma K_{3} w_{3}\left(h_{2}, \widetilde{\lambda}\right) \\
& =K_{2} \gamma w_{3}\left(h_{2}, \widetilde{\lambda}\right)-K_{3} \gamma w_{3}\left(h_{2}, \widetilde{\lambda}\right) \\
& =\gamma\left(K_{2}-K_{3}\right) w_{3}\left(h_{2}, \widetilde{\lambda}\right)=0 .
\end{aligned}
$$

Since $\gamma\left(K_{2}-K_{3}\right) \neq 0$ it follows that

$$
w_{3}\left(h_{2}, \widetilde{\lambda}\right)=0 .
$$

By the same procedure from equality (7) we can derive that

$$
w_{3}^{\prime}\left(h_{2}, \widetilde{\lambda}\right)=0 .
$$

From the fact that $w_{3}(x, \tilde{\lambda})$ is a solution of the differential (1) on $\left[h_{2}, \pi\right]$ and satisfies the initial conditions (21) and (22), it follows that $w_{3}(x, \tilde{\lambda})=0$ identically on $\left[h_{2}, \pi\right]$.

By using this method, we may also find

$$
\begin{aligned}
& w_{2}\left(h_{2}, \tilde{\lambda}\right)=w_{2}^{\prime}\left(h_{2}, \tilde{\lambda}\right)=0, \\
& w_{1}\left(h_{1}, \tilde{\lambda}\right)=w_{1}^{\prime}\left(h_{1}, \tilde{\lambda}\right)=0 .
\end{aligned}
$$

From the latter discussions of $w_{3}(x, \tilde{\lambda})$, it follows that $w_{2}$ $(x, \tilde{\lambda})=0$ and $w_{1}(x, \tilde{\lambda})=0$ identically on $\left(h_{1}, h_{2}\right)$ and $\left[0, h_{1}\right)$, but this contradicts (10), thus completing the proof.

\section{An Existence Theorem}

The function $w(x, \lambda)$ defined in Section 1 is a nontrivial solution of (1) satisfying conditions (2) and (4)-(7). Putting $w(x, \lambda)$ into (3), we get the characteristic equation

$$
F(\lambda) \equiv w(\pi, \lambda) \cos \beta+w^{\prime}(\pi, \lambda) \sin \beta=0 .
$$

By Theorem 2 the set of eigenvalues of boundary-value problem (1)-(7) coincides with the set of real roots of (24). Let

$$
\begin{gathered}
q_{1}=\int_{0}^{h_{1}}|q(\tau)| d \tau, \quad q_{2}=\int_{h_{1}}^{h_{2}}|q(\tau)| d \tau, \\
q_{3}=\int_{h_{2}}^{\pi}|q(\tau)| d \tau .
\end{gathered}
$$

Lemma 3. (1) Let $\lambda \geq 4 q_{1}^{2}$. Then for the solution $w_{1}(x, \lambda)$ of (14), the following inequality holds:

$$
\left|w_{1}(x, \lambda)\right| \leq \frac{1}{q_{1}} \sqrt{4 q_{1}^{2} \sin ^{2} \alpha+\cos ^{2} \alpha}, \quad x \in\left[0, h_{1}\right] .
$$

(2) Let $\lambda \geq \max \left\{4 q_{1}^{2}, 4 q_{2}^{2}\right\}$. Then for the solution $w_{2}(x, \lambda)$ of (15), the following inequality holds:

$$
\left|w_{2}(x, \lambda)\right| \leq \frac{4}{q_{1}|\delta|} \sqrt{4 q_{1}^{2} \sin ^{2} \alpha+\cos ^{2} \alpha}, \quad x \in\left[h_{1}, h_{2}\right] .
$$

(3) Let $\lambda \geq \max \left\{4 q_{1}^{2}, 4 q_{2}^{2}, 4 q_{3}^{2}\right\}$. Then for the solution $w_{3}(x, \lambda)$ of $(16)$, the following inequality holds:

$$
\left|w_{3}(x, \lambda)\right| \leq \frac{16}{q_{1}|\delta \gamma|} \sqrt{4 q_{1}^{2} \sin ^{2} \alpha+\cos ^{2} \alpha}, \quad x \in\left[h_{2}, \pi\right] .
$$

Proof. Let $B_{1 \lambda}=\max _{\left[0, h_{1}\right]}\left|w_{1}(x, \lambda)\right|$. Then from (14), it follows that, for every $\lambda>0$, the following inequality holds:

$$
B_{1 \lambda} \leq \sqrt{\sin ^{2} \alpha+\frac{\cos ^{2} \alpha}{s^{2}}}+\frac{1}{s} B_{1 \lambda} q_{1} .
$$

If $s \geq 2 q_{1}$ we get (26). Differentiating (14) with respect to $x$, we have

$$
\begin{aligned}
w_{1}^{\prime}(x, \lambda)= & -s \sin \alpha \sin s x-\cos \alpha \cos s x \\
& -\int_{0}^{x} q(\tau) \cos s(x-\tau) w_{1}(\tau-\Delta(\tau), \lambda) d \tau .
\end{aligned}
$$

From expressions of (30) and (26), it follows that, for $s \geq 2 q_{1}$, the following inequality holds:

$$
\frac{\left|w_{1}^{\prime}(x, \lambda)\right|}{s} \leq \frac{1}{q_{1}} \sqrt{4 q_{1}^{2} \sin ^{2} \alpha+\cos ^{2} \alpha} .
$$

Let $B_{2 \lambda}=\max _{\left[h_{1}, h_{2}\right]}\left|w_{2}(x, \lambda)\right|$. Then from (11), (26), and (31) it follows that, for $s \geq 2 q_{1}$ and $s \geq 2 q_{2}$, the following inequality holds:

$$
B_{2 \lambda} \leq \frac{2}{q_{1}|\delta|} \sqrt{4 q_{1}^{2} \sin ^{2} \alpha+\cos ^{2} \alpha}+\frac{1}{2 q_{2}} B_{2 \lambda} q_{2} .
$$

Hence, if $\lambda \geq \max \left\{4 q_{1}^{2}, 4 q_{2}^{2}\right\}$, it reduces to (27). Differentiating (15) with respect to, we get

$$
\begin{aligned}
w_{2}^{\prime}(x, \lambda)= & -\frac{s}{\delta} w_{1}\left(h_{1}, \lambda\right) \sin s\left(x-h_{1}\right) \\
& +\frac{w_{1}^{\prime}\left(h_{1}, \lambda\right)}{\delta} \cos s\left(x-h_{1}\right) \\
& -\int_{h_{1}}^{x} q(\tau) \cos s(x-\tau) w_{2}(\tau-\Delta(\tau), \lambda) d \tau . \\
& (s=\sqrt{\lambda}, \lambda>0) .
\end{aligned}
$$


From (26) and (33), it follows that, for $s \geq 2 q_{1}$ and $s \geq 2 q_{2}$, the following inequality holds:

$$
\frac{\left|w_{2}^{\prime}(x, \lambda)\right|}{s} \leq \frac{4}{|\delta| q_{1}} \sqrt{4 q_{1}^{2} \sin ^{2} \alpha+\cos ^{2} \alpha} .
$$

Let $B_{3 \lambda}=\max _{\left[h_{2}, \pi\right]}\left|w_{3}(x, \lambda)\right|$. Then from (16), (27), and (34) it follows that, for $s \geq 2 q_{1}, s \geq 2 q_{2}$ and $s \geq 2 q_{3}$, the following inequality holds:

$$
B_{3 \lambda} \leq \frac{8}{q_{1}|\delta \gamma|} \sqrt{4 q_{1}^{2} \sin ^{2} \alpha+\cos ^{2} \alpha}+\frac{1}{s} B_{3 \lambda} q_{3} .
$$

Hence, if $\lambda \geq \max \left\{4 q_{1}^{2}, 4 q_{2}^{2}, 4 q_{3}^{2}\right\}$ we procure (28).

Theorem 4. The problem (1)-(7) has an infinite set of positive eigenvalues.

Proof. Differentiating (16) with respect to $x$, we readily see that

$$
\begin{aligned}
w_{3}^{\prime}(x, \lambda)= & -\frac{s}{\gamma} w_{2}\left(h_{2}, \lambda\right) \sin s\left(x-h_{2}\right) \\
& +\frac{w_{2}^{\prime}\left(h_{2}, \lambda\right)}{\gamma} \cos s\left(x-h_{2}\right) \\
& -\int_{h_{2}}^{x} q(\tau) \cos s(x-\tau) w_{3}(\tau-\Delta(\tau), \lambda) d \tau . \\
& (s=\sqrt{\lambda}, \lambda>0) .
\end{aligned}
$$

With the helps of (14), (15), (16), (24), (30), and (36), we have the following:

$$
\begin{aligned}
& {\left[\frac { 1 } { \gamma } \left\{\frac { 1 } { \delta } \left(\sin \alpha \cos s h_{1}-\frac{\cos \alpha}{s} \sin s h_{1}\right.\right.\right.} \\
& \left.\quad-\frac{1}{s} \int_{0}^{h_{1}} q(\tau) \sin s\left(h_{1}-\tau\right) w_{1}(\tau-\Delta(\tau), \lambda) d \tau\right) \\
& \quad \times \cos s\left(h_{2}-h_{1}\right) \\
& \quad-\frac{1}{s \delta}\left(s \sin \alpha \sin s h_{1}+\cos \alpha \cos s h_{1}\right. \\
& \quad \times \sin s\left(h_{2}-h_{1}\right) \\
& \left.\quad-\frac{1}{s} \int_{h_{1}}^{h_{2}} q(\tau) \sin s\left(h_{2}-\tau\right) w_{2}(\tau-\Delta(\tau), \lambda) d \tau\right\}
\end{aligned}
$$

$$
\begin{aligned}
& \times \cos s\left(\pi-h_{2}\right) \\
& +\frac{1}{s \gamma}\left\{-\frac{s}{\delta}\left(\sin \alpha \cos s h_{1}-\frac{\cos \alpha}{s} \sin s h_{1}\right.\right. \\
& -\frac{1}{s} \int_{0}^{h_{1}} q(\tau) \sin s\left(h_{1}-\tau\right) \\
& \left.\times w_{1}(\tau-\Delta(\tau), \lambda) d \tau\right) \\
& \times \sin s\left(h_{2}-h_{1}\right) \\
& +\frac{1}{\delta}\left(-s \sin \alpha \sin s h_{1}-\cos \alpha \cos s h_{1}\right. \\
& -\int_{0}^{h_{1}} q(\tau) \cos s\left(h_{1}-\tau\right) \\
& \left.\times w_{1}(\tau-\Delta(\tau), \lambda) d \tau\right) \\
& \times \cos s\left(h_{2}-h_{1}\right) \\
& \left.-\int_{h_{1}}^{h_{2}} q(\tau) \cos s\left(h_{2}-\tau\right) w_{2}(\tau-\Delta(\tau), \lambda) d \tau\right\} \\
& \times \sin s\left(\pi-h_{2}\right) \\
& \left.-\frac{1}{s} \int_{h_{2}}^{\pi} q(\tau) \sin s(\pi-\tau) w_{3}(\tau-\Delta(\tau), \lambda) d \tau\right] \cos \beta \\
& +\left[-\frac{s}{\gamma}\left\{\frac { 1 } { \delta } \left(\sin \alpha \cos s h_{1}-\frac{\cos \alpha}{s} \sin s h_{1}\right.\right.\right. \\
& -\frac{1}{s} \int_{0}^{h_{1}} q(\tau) \sin s\left(h_{1}-\tau\right) \\
& \left.\times w_{1}(\tau-\Delta(\tau), \lambda) d \tau\right) \\
& \times \cos s\left(h_{2}-h_{1}\right) \\
& -\frac{1}{s \delta}\left(s \sin \alpha \sin s h_{1}+\cos \alpha \cos s h_{1}\right. \\
& +\int_{0}^{h_{1}} q(\tau) \cos s\left(h_{1}-\tau\right) \\
& \left.\times w_{1}(\tau-\Delta(\tau), \lambda) d \tau\right) \\
& \times \sin s\left(h_{2}-h_{1}\right) \\
& \left.-\frac{1}{s} \int_{h_{1}}^{h_{2}} q(\tau) \sin s\left(h_{2}-\tau\right) w_{2}(\tau-\Delta(\tau), \lambda)\right\} \\
& \times \sin s\left(\pi-h_{2}\right)
\end{aligned}
$$




$$
\begin{aligned}
& +\frac{1}{\gamma}\left\{-\frac{s}{\delta} \times\left(\sin \alpha \cos s h_{1}-\frac{\cos \alpha}{s} \sin s h_{1}\right.\right. \\
& -\frac{1}{s} \int_{0}^{h_{1}} q(\tau) \sin s\left(h_{1}-\tau\right) \\
& \left.\times w_{1}(\tau-\Delta(\tau), \lambda) d \tau\right) \\
& \times \sin s\left(h_{2}-h_{1}\right) \\
& +\frac{1}{\delta}\left(-s \sin \alpha \sin s h_{1}-\cos \alpha \cos s h_{1}\right. \\
& -\int_{0}^{h_{1}} q(\tau) \cos s\left(h_{1}-\tau\right) \\
& \left.\times w_{1}(\tau-\Delta(\tau), \lambda) d \tau\right) \\
& \quad \times \cos s\left(h_{2}-h_{1}\right) \\
& \left.-\int_{h_{1}}^{h_{2}} q(\tau) \cos s\left(h_{2}-\tau\right) w_{2}(\tau-\Delta(\tau), \lambda) d \tau\right\} \\
& \times \cos s\left(\pi-h_{2}\right) \\
& \left.-\int_{h_{2}}^{\pi} q(\tau) \cos s(\pi-\tau) w_{3}(\tau-\Delta(\tau), \lambda) d \tau\right] \sin \beta=0 .
\end{aligned}
$$

Let $\lambda$ be sufficiently big. Then, by (26), (27), and (28), (37) may be rewritten in the following form:

$$
\begin{aligned}
-\frac{s \sin \alpha \sin \beta}{\gamma \delta}\{ & \cos s h_{2} \sin s\left(\pi-h_{2}\right) \\
+ & \left.\sin s h_{2} \cos s\left(\pi-h_{2}\right)\right\}+O(1)=0, \\
& s \sin s \pi+O(1)=0 .
\end{aligned}
$$

Obviously, for big $s$ (39) has an infinite set of roots. Thus, the proof of the theorem is completed.

\section{Asymptotic Formulas for Eigenvalues and Eigenfunctions}

Now we begin to study asymptotic properties of eigenvalues and eigenfunctions. In the following we will assume that is sufficiently big. From (14) and (26), we obtain

$$
w_{1}(x, \lambda)=O(1) \text { on }\left[0, h_{1}\right] .
$$

By (15) and (27), this leads to

$$
w_{2}(x, \lambda)=O(1) \quad \text { on }\left[h_{1}, h_{2}\right] .
$$

By (16) and (28), this leads to

$$
w_{3}(x, \lambda)=O(1) \quad \text { on }\left[h_{2}, \pi\right] .
$$

The existence and continuity of the derivatives $w_{1 s}^{\prime}(x, \lambda)$ for $0 \leq x \leq h_{1},|\lambda|<\infty, w_{2 s}^{\prime}(x, \lambda)$ for $h_{1} \leq x \leq h_{2},|\lambda|<\infty$ and $w_{3 s}^{\prime}(x, \lambda)$ for $h_{2} \leq x \leq \pi,|\lambda|<\infty$ follows from Theorem 1.4.1 in [5].
Lemma 5. The following holds true:

$$
\begin{array}{ll}
w_{1 s}^{\prime}(x, \lambda)=O(1), & x \in\left[0, h_{1}\right], \\
w_{2 s}^{\prime}(x, \lambda)=O(1), & x \in\left[h_{1}, h_{2}\right], \\
w_{3 s}^{\prime}(x, \lambda)=O(1), & x \in\left[h_{2}, \pi\right] .
\end{array}
$$

Proof. By differentiating (16) with respect to $s$, we get, by (43) and (44) the following:

$$
\begin{array}{r}
w_{3 s}^{\prime}(x, \lambda) \\
=-\frac{1}{s} \int_{h_{2}}^{x} q(\tau) \sin s(x-\tau) w_{3 s}^{\prime}(\tau-\Delta(\tau), \lambda)+Z(x, \lambda), \\
\left(|Z(x, \lambda)| \leq Z_{0}\right) .
\end{array}
$$

Let $D_{\lambda}=\max _{\left[h_{2}, \pi\right]}\left|w_{3 s}^{\prime}(x, \lambda)\right|$. Then the existence of $D_{\lambda}$ follows from continuity of derivation for $x \in\left[h_{2}, \pi\right]$. From (46)

$$
D_{\lambda} \leq \frac{1}{s} q_{3} D_{\lambda}+Z_{0} .
$$

Now let $s \geq 2 q_{3}$. Then $D_{\lambda} \leq 2 Z_{0}$ and the validity of the asymptotic formula (45) follows. Formulas (43) and (44) may be proved analogically.

Theorem 6. Let $n$ be a natural number. For each sufficiently big $n$ there is exactly one eigenvalue of the problem (1)-(7) near $n^{2}$.

Proof. We consider the expression which is denoted by $O(1)$ in (39). If formulas (40)-(45) are taken into consideration, it can be shown by differentiation with respect to $s$ that for big $s$ this expression has bounded derivative. We will show that, for big $n$, only one root (39) lies near to each $n$. We consider the function $\phi(s)=s \sin s \pi+O(1)$. Its derivative, which has the form $\phi^{\prime}(s)=\sin s \pi+s \pi \cos s \pi+O(1)$, does not vanish for $s$ close to $n$ for sufficiently big $n$. Thus our assertion follows by Rolle's Theorem.

Let $n$ be sufficiently big. In what follows we will denote by $\lambda_{n}=s_{n}^{2}$ the eigenvalue of the problem (1)-(7) situated near $n^{2}$. We set $s_{n}=n+\delta_{n}$. Then from (39) it follows that $\delta_{n}=O(1 / n)$. Consequently

$$
\begin{aligned}
& s_{n}=n+O\left(\frac{1}{n}\right), \\
& \lambda_{n}=n^{2}+O(1) .
\end{aligned}
$$

Formula (48) makes it possible to obtain asymptotic expressions for eigenfunction of the problem (1)-(7). From (14), (30), and (40), we get

$$
\begin{aligned}
& w_{1}(x, \lambda)=\sin \alpha \cos s x+O\left(\frac{1}{s}\right), \\
& w_{1}^{\prime}(x, \lambda)=-s \sin \alpha \sin s x+O(1) .
\end{aligned}
$$


From expressions of (15), (31), (39), and (41), we easily see that

$$
\begin{aligned}
& w_{2}(x, \lambda)=\frac{\sin \alpha}{\delta} \cos s x+O\left(\frac{1}{s}\right) \\
& w_{3}(x, \lambda)=\frac{\sin \alpha}{\delta \gamma} \cos s x+O\left(\frac{1}{s}\right)
\end{aligned}
$$

By substituting (48) into (50), (52), we find that

$$
\begin{aligned}
& u_{1 n}=w_{1}\left(x, \lambda_{n}\right)=\sin \alpha \cos n x+O\left(\frac{1}{n}\right), \\
& u_{2 n}=w_{2}\left(x, \lambda_{n}\right)=\frac{\sin \alpha}{\delta} \cos n x+O\left(\frac{1}{n}\right), \\
& u_{3 n}=w_{3}\left(x, \lambda_{n}\right)=\frac{\sin \alpha}{\delta \gamma} \cos n x+O\left(\frac{1}{n}\right) .
\end{aligned}
$$

Hence the eigenfunctions $u_{n}(x)$ have the following asymptotic representation:

$$
u_{n}(x)= \begin{cases}\sin \alpha \cos n x+O\left(\frac{1}{n}\right), & \text { for } x \in\left[0, h_{1}\right), \\ \frac{\sin \alpha}{\delta} \cos n x+O\left(\frac{1}{n}\right), & \text { for } x \in\left(h_{1}, h_{2}\right), \\ \frac{\sin \alpha}{\delta \gamma} \cos n x+O\left(\frac{1}{n}\right), & \text { for } x \in\left(h_{2}, \pi\right] .\end{cases}
$$

Under some additional conditions the more exact asymptotic formulas which depend upon the retardation may be obtained. Let us assume that the following conditions are fulfilled.

(a) The derivatives $q^{\prime}(x)$ and $\Delta^{\prime \prime}(x)$ exist and are bounded in $\left[0, h_{1}\right) \cup\left(h_{1}, h_{2}\right) \cup\left(h_{2}, \pi\right]$ and have finite limits $q^{\prime}\left(h_{1} \pm 0\right)=$ $\lim _{x \rightarrow h_{1} \pm 0} q^{\prime}(x), q^{\prime}\left(h_{2} \pm 0\right)=\lim _{x \rightarrow h_{2} \pm 0} q^{\prime}(x)$ and $\Delta^{\prime \prime}\left(h_{1} \pm 0\right)=$ $\lim _{x \rightarrow h_{1} \pm 0} \Delta^{\prime \prime}(x), \Delta^{\prime \prime}\left(h_{2} \pm 0\right)=\lim _{x \rightarrow h_{2} \pm 0} \Delta^{\prime \prime}(x)$, respectively.

(b) $\Delta^{\prime}(x) \leq 1$ in $\left[0, h_{1}\right) \cup\left(h_{1}, h_{2}\right) \cup\left(h_{2}, \pi\right], \Delta(0)=0$, $\lim _{x \rightarrow h_{1}+0} \Delta(x)=0$, and $\lim _{x \rightarrow h_{2}+0} \Delta(x)=0$.

It is easy to see that, using (b)

$$
\begin{gathered}
x-\Delta(x) \geq 0, \quad x \in\left[0, h_{1}\right), \\
x-\Delta(x) \geq h_{1}, \quad x \in\left(h_{1}, h_{2}\right), \\
x-\Delta(x) \geq h_{2}, \quad x \in\left(h_{2}, \pi\right]
\end{gathered}
$$

are obtained.

By (50), (52), and (55), we have

$$
\begin{aligned}
& w_{1}(\tau-\Delta(\tau), \lambda)=\sin \alpha \cos s(\tau-\Delta(\tau))+O\left(\frac{1}{s}\right), \\
& w_{2}(\tau-\Delta(\tau), \lambda)=\frac{\sin \alpha}{\delta} \cos s(\tau-\Delta(\tau))+O\left(\frac{1}{s}\right), \\
& w_{3}(\tau-\Delta(\tau), \lambda)=\frac{\sin \alpha}{\delta \gamma} \cos s(\tau-\Delta(\tau))+O\left(\frac{1}{s}\right)
\end{aligned}
$$

on $\left[0, h_{1}\right),\left(h_{1}, h_{2}\right)$ and $\left(h_{2}, \pi\right]$, respectively.
Under the conditions (a) and (b) the following formulas:

$$
\begin{aligned}
& \int_{0}^{x} q(\tau) \cos s(2 \tau-\Delta(\tau)) d \tau=O\left(\frac{1}{s}\right), \\
& \int_{0}^{x} q(\tau) \sin s(2 \tau-\Delta(\tau)) d \tau=O\left(\frac{1}{s}\right)
\end{aligned}
$$

can be proved by the same technique in Lemma 3.3.3 in [5].

Putting the expressions (56), (57), and (58) into (37), and then using (59), after long operations we have

$$
\begin{aligned}
& \frac{\cos s \pi \sin (\alpha-\beta)-s \sin \alpha \sin \beta \sin s \pi}{\delta \gamma} \\
& -\frac{\sin \alpha \sin \beta}{2 \delta \gamma} \int_{0}^{\pi} q(\tau) \cos s(\pi-\Delta(\tau)) d \tau \\
& +O\left(\frac{1}{s}\right)=0 .
\end{aligned}
$$

Hence

$$
\begin{aligned}
\tan s \pi=\frac{1}{s}\left(\frac{\sin (\alpha-\beta)}{\sin \alpha \sin \beta}\right. \\
\left.\quad-\frac{1}{2} \int_{0}^{\pi} q(\tau) \cos s(\pi-\Delta(\tau)) d \tau\right) \\
+O\left(\frac{1}{s^{2}}\right)
\end{aligned}
$$

Again, if we take $s_{n}=n+\delta_{n}$, then from (48)

$$
\begin{aligned}
\tan \left(\left(n+\delta_{n}\right) \pi\right)= & \tan \delta_{n} \pi \\
= & \frac{1}{n}\left(\frac{\sin (\alpha-\beta)}{\sin \alpha \sin \beta}\right. \\
& \left.\quad-\frac{1}{2} \int_{0}^{\pi} q(\tau) \cos s(\pi-\Delta(\tau)) d \tau\right) \\
& +O\left(\frac{1}{n^{2}}\right) .
\end{aligned}
$$

Hence for big $n$,

$$
\begin{aligned}
\delta_{n}= & \frac{1}{n \pi}\left(\frac{\sin (\alpha-\beta)}{\sin \alpha \sin \beta}-\frac{1}{2} \int_{0}^{\pi} q(\tau) \cos s(\pi-\Delta(\tau)) d \tau\right) \\
& +O\left(\frac{1}{n^{2}}\right)
\end{aligned}
$$

and finally

$$
\begin{aligned}
s_{n}= & n+\frac{1}{n \pi}\left(\frac{\sin (\alpha-\beta)}{\sin \alpha \sin \beta}-\frac{1}{2} \int_{0}^{\pi} q(\tau) \cos s(\pi-\Delta(\tau)) d \tau\right) \\
& +O\left(\frac{1}{n^{2}}\right) .
\end{aligned}
$$

Thus, we have proven the following theorem. 
Theorem 7. If conditions (a) and (b) are satisfied, then the eigenvalues $\lambda_{n}=s_{n}^{2}$ of the problem (1)-(7) have the (64) asymptotic formula for $n \rightarrow \infty$.

Now, we may obtain sharper asymptotic formulas for the eigenfunctions. From (14), (56), and (59), we have

$$
\begin{aligned}
w_{1}(x, \lambda)= & \sin \alpha \cos s x\left[1+\frac{1}{2 s} \int_{0}^{x} q(\tau) \sin s \Delta(\tau) d \tau\right] \\
& -\frac{\sin s x}{s}\left[\cos \alpha+\frac{\sin \alpha}{2} \int_{0}^{x} q(\tau) \cos s \Delta(\tau) d \tau\right] \\
& +O\left(\frac{1}{s^{2}}\right), \quad x \in\left[0, h_{1}\right) .
\end{aligned}
$$

Now, replacing $s$ by $s_{n}$ and using (64), we have

$$
\begin{aligned}
& u_{1 n}(x) \\
& =w_{1}\left(x, \lambda_{n}\right) \\
& =\sin \alpha\left\{\cos n x\left[1+\frac{1}{2 n} \int_{0}^{x} q(\tau) \sin (n \Delta(\tau)) d \tau\right]\right. \\
& -\frac{\sin n x}{n \pi}\left[\left(\frac{\sin (\alpha-\beta)}{\sin \alpha \sin \beta}\right.\right. \\
& \left.\quad-\frac{1}{2} \int_{0}^{\pi} q(\tau) \cos (n(\pi-\Delta(\tau))) d \tau\right) x \\
& \left.\left.+O\left(\frac{1}{n^{2}}\right) \cdot \quad+\left(\cot \alpha+\frac{1}{2} \int_{0}^{x} q(\tau) \cos (n \Delta(\tau)) d \tau\right) \pi\right]\right\}
\end{aligned}
$$

From (15), (57), (59), and (65) we have

$$
\begin{aligned}
w_{2}(x, \lambda)= & \frac{\sin \alpha \cos s x}{\delta}\left[1+\frac{1}{2 s} \int_{0}^{x} q(\tau) \sin s \Delta(\tau) d \tau\right] \\
& -\frac{\sin s x}{s \delta}\left[\cos \alpha+\frac{\sin \alpha}{2} \int_{0}^{x} q(\tau) \cos s \Delta(\tau) d \tau\right] \\
& +O\left(\frac{1}{s^{2}}\right), \quad x \in\left(h_{1}, h_{2}\right) .
\end{aligned}
$$

Now, replacing $s$ by $s_{n}$ and using (64), we have

$$
\begin{aligned}
& u_{2 n}(x) \\
& \quad=w_{2}\left(x, \lambda_{n}\right) \\
& \quad=\frac{\sin \alpha}{\delta}\left\{\cos n x\left[1+\frac{1}{2 n} \int_{0}^{x} q(\tau) \sin (n \Delta(\tau)) d \tau\right]\right.
\end{aligned}
$$

$$
\begin{aligned}
& -\frac{\sin n x}{n \pi}\left[\left(\frac{\sin (\alpha-\beta)}{\sin \alpha \sin \beta}\right.\right. \\
& \left.-\frac{1}{2} \int_{0}^{\pi} q(\tau) \cos (n(\pi-\Delta(\tau))) d \tau\right) x \\
& \left.\left.+\left(\cot \alpha+\frac{1}{2} \int_{0}^{x} q(\tau) \cos (n \Delta(\tau)) d \tau\right) \pi\right]\right\} \\
& +O\left(\frac{1}{n^{2}}\right) .
\end{aligned}
$$

From (16), (58), (59), (65), and (67) and after long operations, we have

$$
\begin{aligned}
w_{3}(x, \lambda)= & \frac{\sin \alpha \cos s x}{\delta \gamma}\left[1+\frac{1}{2 s} \int_{0}^{x} q(\tau) \sin s \Delta(\tau) d \tau\right] \\
& -\frac{\sin s x}{s \delta \gamma}\left[\cos \alpha+\frac{\sin \alpha}{2} \int_{0}^{x} q(\tau) \cos s \Delta(\tau) d \tau\right] \\
& +O\left(\frac{1}{s^{2}}\right), \quad x \in\left(h_{2}, \pi\right] .
\end{aligned}
$$

Now replacing $s$ by $s_{n}$ and using (64), we have

$$
\begin{aligned}
& u_{3 n}(x) \\
& =w_{3}\left(x, \lambda_{n}\right) \\
& =\frac{\sin \alpha}{\delta \gamma}\left\{\cos n x\left[1+\frac{1}{2 n} \int_{0}^{x} q(\tau) \sin (n \Delta(\tau)) d \tau\right]\right. \\
& -\frac{\sin n x}{n \pi}\left[\left(\frac{\sin (\alpha-\beta)}{\sin \alpha \sin \beta}\right.\right. \\
& \left.\quad+\frac{1}{2} \int_{0}^{\pi} q(\tau) \cos (n(\pi-\Delta(\tau))) d \tau\right) x \\
& +O\left(\frac{1}{n^{2}}\right) .
\end{aligned}
$$

Thus, we have proven the following theorem.

Theorem 8. If conditions (a) and (b) are satisfied, then the eigenfunctions $u_{n}(x)$ of the problem (1)-(7) have the following asymptotic formula for $n \rightarrow \infty$ :

$$
u_{n}(x)= \begin{cases}u_{1 n}(x), & x \in\left[0, h_{1}\right), \\ u_{2 n}(x), & x \in\left(h_{1}, h_{2}\right), \\ u_{3 n}(x), & x \in\left(h_{2}, \pi\right],\end{cases}
$$

where $u_{1 n}(x), u_{2 n}(x)$, and $u_{3 n}(x)$ are determined as in (49), (68), and (70), respectively. 


\section{References}

[1] G. Teschl, Ordinary Differential Equations and Dynamical Systems, vol. 140, American Mathematical Society, Providence, RI, USA, 2012.

[2] M. Shahriari, A. J. Akbarfam, and G. Teschl, "Uniqueness for inverse Sturm-Liouville problems with a finite number of transmission conditions," Journal of Mathematical Analysis and Applications, vol. 395, no. 1, pp. 19-29, 2012.

[3] T. Kim, "Identities involving Frobenius-Euler polynomials arising from non-linear differential equations," Journal of Number Theory, vol. 132, no. 12, pp. 2854-2865, 2012.

[4] S. B. Norkin, "On a boundary problem of Sturm-Liouville type for a second-order differential equation with a retarded argument," Izvestija Vysših Učebnyh Zavedenii Matematika, vol. 6, no. 7, pp. 203-214, 1958 (Russian).

[5] S. B. Norkin, Differential Equations of the Second Order with Retarded Argument, vol. 31 of Translations of Mathematical Monographs, American Mathematical Society, Providence, RI, USA, 1972.

[6] R. Bellman and K. L. Cooke, Differential-Difference Equations, Academic Press, London, UK, 1963.

[7] G. V. Demidenko and V. A. Likhoshvaĭ, "On differential equations with retarded argument," Siberian Mathematical Journal, vol. 46, no. 3, pp. 417-430, 2005.

[8] G. V. Demidenko and I. I. Matveeva, "On numerical study of asymptotic stability of solutions to linear periodic differential equations with a parameter," Journal of Computational Mathematics and Optimization, vol. 5, no. 3, pp. 163-173, 2009.

[9] A. Bayramov, S. Öztürk Uslu, and S. Aliskan, "Computation of eigenvalues and eigenfunctions of a discontinuous boundary value problem with retarded argument," Applied Mathematics and Computation, vol. 191, no. 2, pp. 592-600, 2007.

[10] E. Şen, S. Araci, and M. Acikgoz, "Asymptotic behavior of eigenvalues and fundamental solutions of one discontinuous fourthorder boundary value problem," Research Journal of Mathematical and Statistical Sciences, vol. 1, no. 1, pp. 23-32, 2013.

[11] E. Şen and S. Araci, "Computation of Eigenvalues and fundamental solutions of a fourth-order boundary value," Proceedings of the Jangjeon Mathematical Society, vol. 15, no. 4, pp. 455-464, 2012.

[12] E. Şen and A. Bayramov, "On calculation of eigenvalues and eigenfunctions of a Sturm-Liouville type problem with retarded argument which contains a spectral parameter in the boundary condition," Journal of Inequalities and Applications, vol. 2011, article 113, 2011.

[13] E. Şen and A. Bayramov, "On a discontinuous Sturm-Liouville type problem with retarded argument," American Institute of Physics Conference Proceedings, vol. 1389, pp. 1172-1175, 2011.

[14] E. Şen and A. Bayramov, "Calculation of eigenvalues and eigenfunctions of a discontinuous boundary value problem with retarded argument which contains a spectral parameter in the boundary condition," Mathematical and Computer Modelling, vol. 54, no. 11-12, pp. 3090-3097, 2011.

[15] A. Bayramov and E. Şen, "On a Sturm-Liouville type problem with retarded argument," Mathematical Methods in the Applied Sciences, vol. 36, no. 1, pp. 39-48, 2013.

[16] C. T. Fulton, "Two-point boundary value problems with eigenvalue parameter contained in the boundary conditions," Proceedings of the Royal Society of Edinburgh A, vol. 77, no. 3-4, pp. 293-308, 1977.
[17] P. A. Binding, P. J. Browne, and B. A. Watson, "Sturm-Liouville problems with boundary conditions rationally dependent on the eigenparameter II," Journal of Computational and Applied Mathematics, vol. 148, no. 1, pp. 147-168, 2002.

[18] N. Altinisik, M. Kadakal, and O. Sh. Mukhtarov, "Eigenvalues and eigenfunctions of discontinuous Sturm-Liouville problems with eigenparameter-dependent boundary conditions," Acta Mathematica Hungarica, vol. 102, no. 1-2, pp. 159-175, 2004.

[19] N. B. Kerimov and Kh. R. Mamedov, "On a boundary value problem with a spectral parameter in the boundary conditions," Sibirskiŭ Matematicheskiǔ Zhurnal, vol. 40, no. 2, pp. 325-335, 1999.

[20] I. Titeux and Y. Yakubov, "Completeness of root functions for thermal conduction in a strip with piecewise continuous coefficients," Mathematical Models \& Methods in Applied Sciences, vol. 7, no. 7, pp. 1035-1050, 1997. 


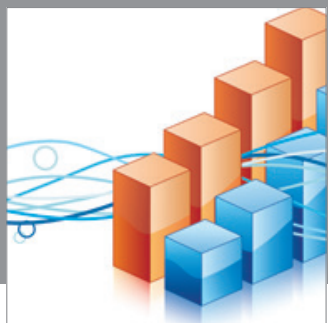

Advances in

Operations Research

mansans

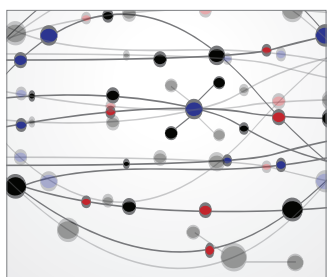

The Scientific World Journal
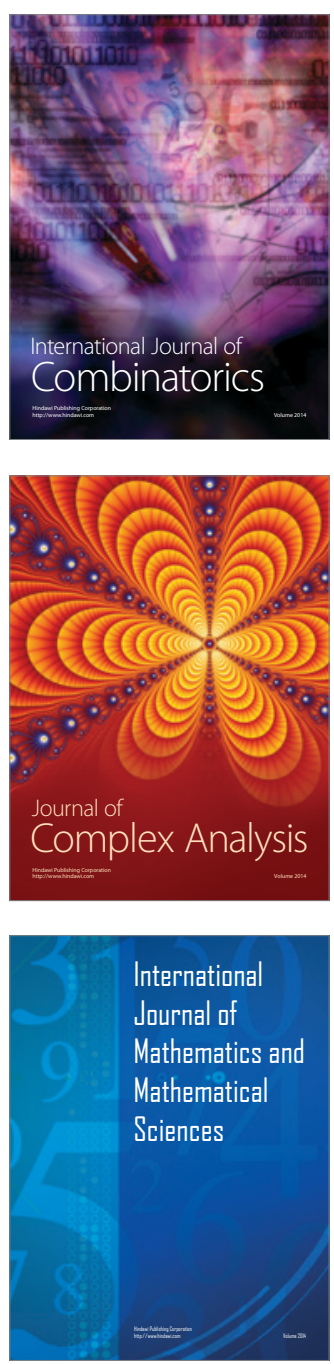
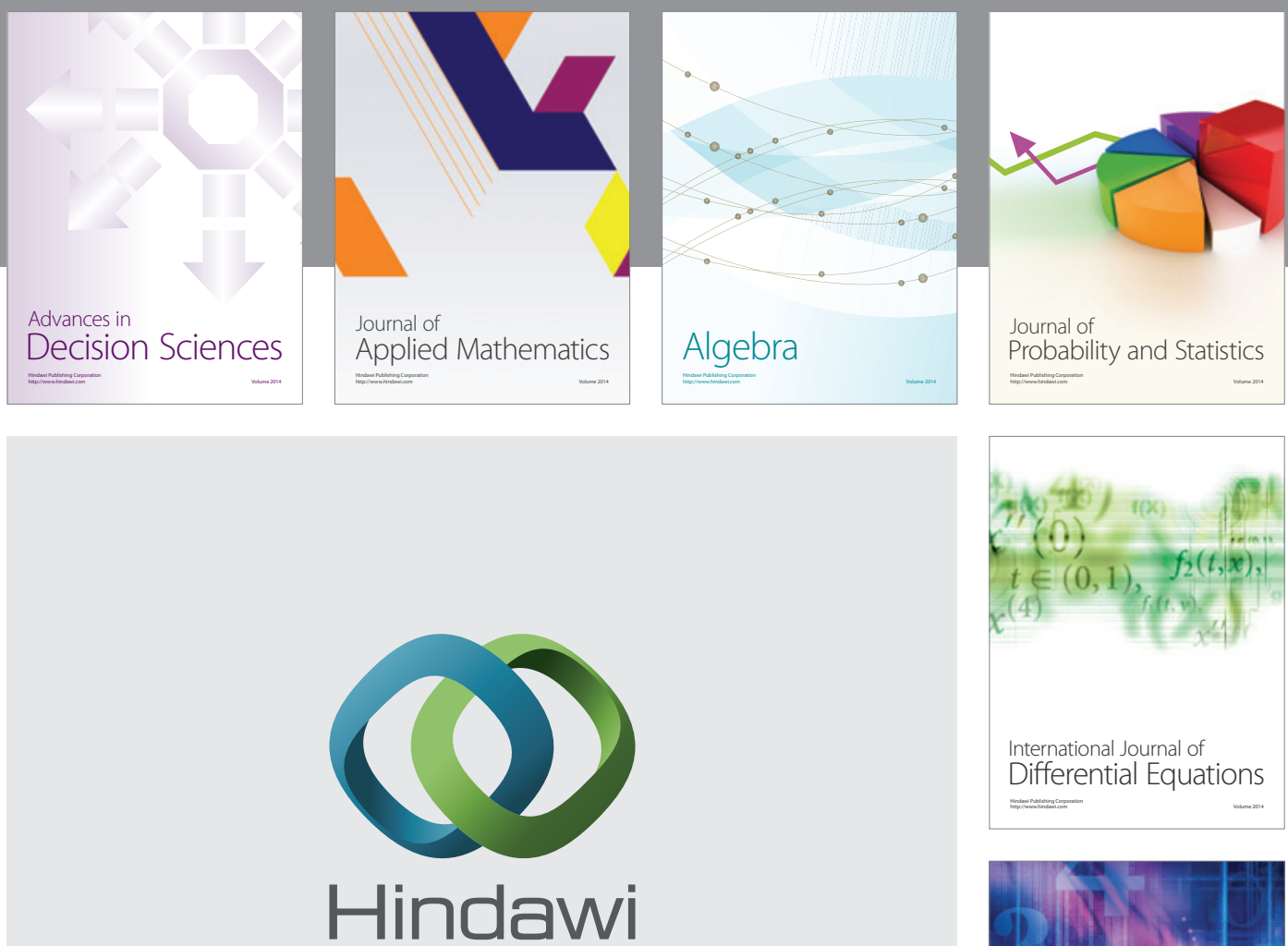

Submit your manuscripts at http://www.hindawi.com
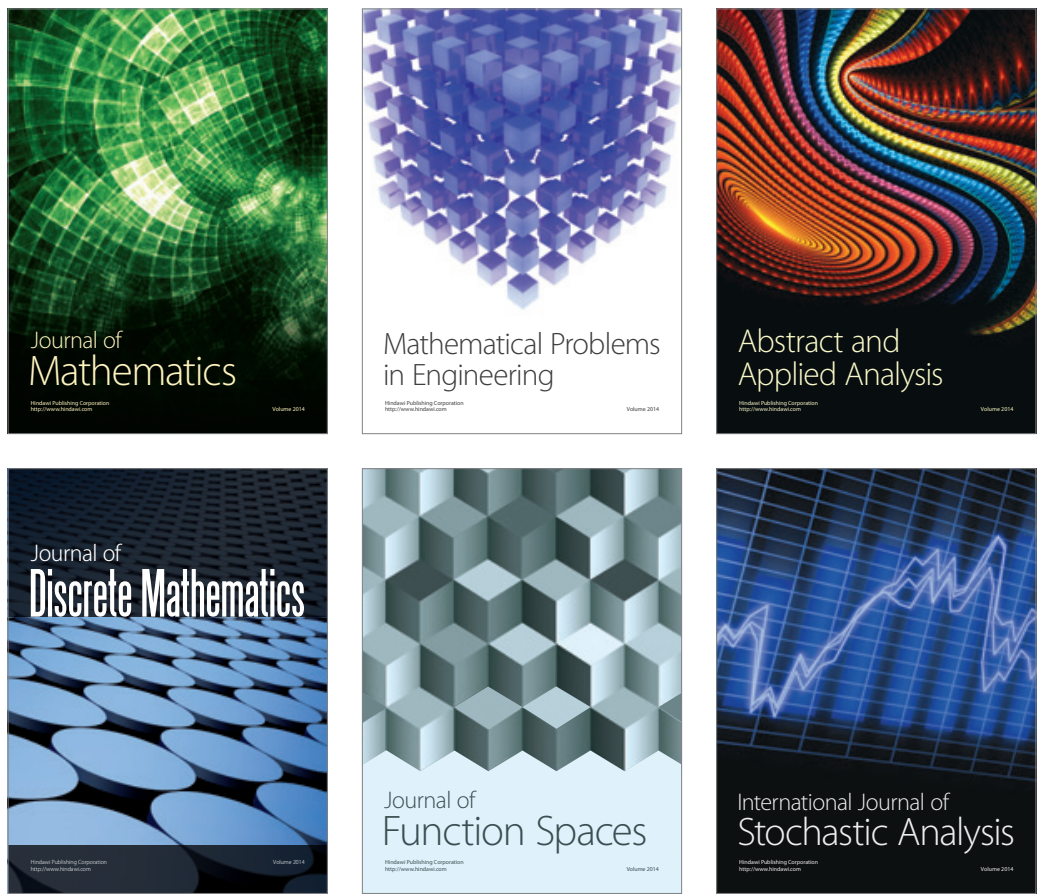

Journal of

Function Spaces

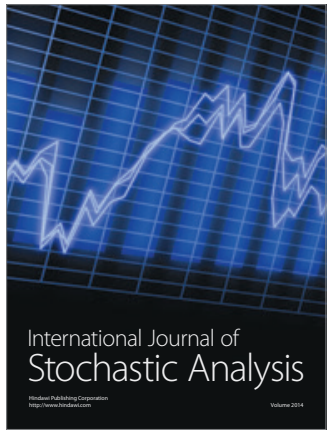

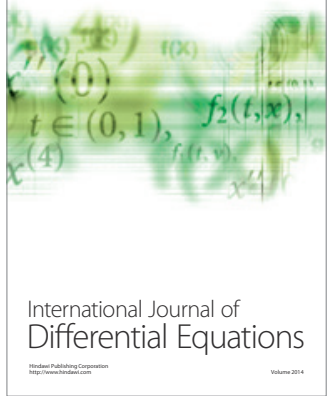
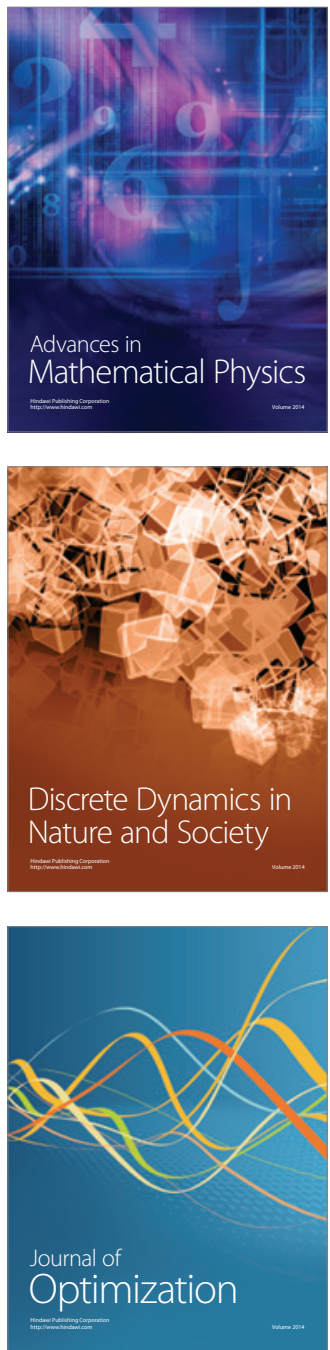